\title{
Visita técnica em unidade de terapia intensiva como método de ensino- aprendizagem na graduação
}

\author{
Technical visit to an intensive care unit as a teaching-learning method in under graduate \\ courses
}
Visita técnica una unidad de cuidados intensivos um método de enseñanza-aprendizaje en la graduacion

Caroline do Nascimento Leite ${ }^{1 *}$, Daniela Vogel de Souza1, Gláucia dos Santos Pereira ${ }^{1}$, Lorena Lourenço Massarra de Lima ${ }^{1}$, Samantha Carvalho dos Mons ${ }^{1}$, Vanessa Alves Bezerra Figueiredo'.

\section{RESUMO}

Objetivo: Relatar a experiência vivenciada por acadêmicas de enfermagem durante as visitas técnicas realizadas em uma unidade de terapia intensiva de um hospital público. Relato de experiência: Trata-se de um relato de experiência das visitas técnicas supervisionadas realizadas através da disciplina de ensino clínico prático em alta complexidade ministrada no 8 o período do curso de graduação em enfermagem de uma instituição de ensino superior, localizada no estado do Rio de Janeiro. Considerações finais: As visitas técnicas podem ser compreendidas como uma ferramenta essencial ao processo de ensino-aprendizagem na graduação. A utilização desse recurso metodológico deve ser estimulada logo após o início do curso uma vez que contribui de forma significativa na formação acadêmica do discente. A oportunidade de realizar as visitas desperta no discente a possibilidade de alinhar conhecimentos teóricos e práticos, colaborando, assim, no processo de construção de um profissional crítico-reflexivo, capaz de propor mudanças e desfechos para os problemas vivenciados bem como melhorias na qualidade da assistência prestada ao cliente.

Palavras-chave: Cuidados críticos, Educação em enfermagem, Ensino.

\begin{abstract}
Objective: To report the experience lived by nursing students during the technical visits under taken in a public hospital intensive care unit. Experience report: This is na experience report of supervised technical visits undertaken in the Practical Clinical Teaching in High Complexity subject taught in the 8th period of the undergraduate nursing course of a higher education institution located in the state of Rio de Janeiro. Final considerations: Technical visits can be understood as an essential tool in the undergraduate teachinglearning process. The use of this methodological resource should be encouraged soon after the beginning of the course as it contributes significantly to the academic formation of the student. The opportunity of holding these visits arouses in the students the possibility of aligning theoretical and practical knowledge, thus collaborating in the construction process of a critical-reflective professional, able to propose changes and outcomes to the problems experienced, as well as improvements in the quality of the assistance provided to the customer.
\end{abstract}

Keywords: Critical care, Nursing education, Teaching.

\section{RESUMEN}

Objetivo: Informar la experiencia vivida por los estudiantes de enfermería durante lasvisitas técnicas realizadas en una unidad de cuidados intensivos de un hospital público.Informe de experiencia: Este es un informe de experiencia de visitas técnicassupervisadas realizadas a través de la asignatura de enseñanza clínica práctica en altacomplejidad que se imparte en el octavo período del curso de pregrado en enfermería

${ }^{1}$ Universidade Estácio de Sá, Rio de Janeiro-RJ. *E-mail: carolnascimento0402@gmail.com 
deuna institución de educación superior ubicada en el estado de Rio de Janeiro.Consideraciones finales:Las visitas técnicas pueden entenderse como una herramienta esencial para el proceso de enseñanzaaprendizaje de pregrado. Se debe alentar el uso de este recurso metodológico poco después del comienzo del curso, ya que contribuye significativamente a la formación académica del estudiante. La oportunidad derealizar las visitas despierta en el alumno la posibilidad de armonizar los conocimientosteóricos y prácticos, colaborando así en el proceso de construcción de un profesionalcrítico-reflexivo, capaz de proponer cambios y resultados a los problemasexperimentados, así como mejoras en la calidad del servicio al cliente.

Palabras clave: Cuidados críticos, Educación en enfermería, Enseñanza.

\section{INTRODUÇÃO}

Com o objetivo de contribuir para a consolidação do Sistema Único de Saúde (SUS) no Brasil, o Ministério da Educação e o Ministério da Saúde instituíram Diretrizes Curriculares Nacionais para 14 profissões da saúde, buscando oferecer uma formação que possibilite a qualificação do cuidado da assistência à saúde, contemplando os princípios do SUS. Tais diretrizes orientam que o processo de formação dos profissionais da saúde deverá ser direcionado ao ensino das competências e habilidades definidas para este profissional, e seu projeto pedagógico, baseado no perfil profissional e nas competências e habilidades desejadas, originando os conteúdos curriculares necessários (BRASIL, 2001; HUMEREZ DC e JANKEVICIUS JV, 2015).

Partindo desse pressuposto, a graduação pode ser entendida como um estágio do processo de formação, que deve ser contínuo para enfrentar a permanente mudança na produção de conhecimentos e uma formação que incorpore os processos de aprender a aprender e que busque atender às demandas da sociedade, além de priorizar uma formação de profissionais autônomos e flexíveis (COSTA DAS, et al., 2018).

A visita técnica como uma prática curricular hospitalar é fundamental para ambientação dos alunos que estão tendo contato pela primeira vez no campo hospitalar e na complementação da formação como futuros profissionais de enfermagem. Ao conhecer o campo prático estão em busca de colocar em prática o que se aprendeu nas teorias para melhor aperfeiçoamento dos estudos e acompanhar em tempo real as técnicas e as rotinas da equipe de enfermagem (DUEÑAS CVM, et al., 2015).

Durante esse percurso os acadêmicos de enfermagem são estimulados a refletir sobre fatos vividos no ambiente hospitalar, para que no decorrer dessa jornada eles possam desenvolver habilidades. Combinando assim, atributos para domínio cognitivo (saber), habilidades como domínio psicomotor (saber fazer) e atitudes que são domínio afetivo (saber ser e conviver) para adquirir visão ampla do seu campo de atuação (PARANHOS VD e MENDES MMR, 2010).

O processo de ensino não está direcionado somente ao desenvolvimento das habilidades específicas e rotineiras que lhes são solicitadas, ele busca também desenvolver a compreensão dos impasses ocasionados por eventos de ordem interna, que quando combinados com eventos externos ou interpessoais, podem ocasionar ansiedade, em decorrência do desequilíbrio adaptativo da pessoa diante do modo como vivencia o processo (DIAS EP, et al., 2014).

Diante do exposto, o presente estudo fundamenta-se em relatar a experiência vivenciada por acadêmicas de enfermagem, durante visitas técnicas realizadas em unidade de terapia intensiva.

\section{RELATO DA EXPERIÊNCIA}

Relato de experiência de caráter crítico-reflexivo baseado na vivência das acadêmicas de enfermagem durante visitas técnicas realizadas em unidade de terapia intensiva durante o ensino clínico prático em alta complexidade.

A vivência ocorreu em um período de quatro meses, entre os meses de março e junho de 2019. As disciplinas de ensino clínico da instituição são constituídas pela parte teórica e prática. Para cursar a disciplina de alta complexidade, o discente deverá ter cumprido minimamente as disciplinas de Sistematização do Cuidar III e Ensino Clínico em Saúde Mental teórico e prático. 
Sua parte prática é cumprida através da divisão das atividades, mediante cronograma, de forma que 1/3 dessas atividades seja constituído por visitas técnicas supervisionadas em instituições hospitalares e os outros $2 / 3$, em laboratório, onde os discentes têm a oportunidade de empregar as técnicas discutidas na disciplina teórica. Nas visitas técnicas, busca-se alinhar as percepções e reflexões alcançadas com a teoria e a prática, contudo observa- se que em alguns momentos não é possível realizar essa análise, uma vez que o contexto e realidade do SUS e as condições e estrutura da instituição não a permitem.

A unidade visitada foi um hospital público de grande porte localizado no Estado do Rio de Janeiro, que atua sob gestão municipal vinculada a uma organização social de saúde. O setor visitado foi a unidade de terapia intensiva. Esta possui 14 leitos, sendo 2 destes reservados para pacientes em precaução de contato. A equipe de enfermagem é composta por 2 enfermeiros e 6 técnicos de enfermagem, cujo regime de plantão é de 30 horas semanais, distribuídos em escalas de 12 horas por equipe, totalizando 6 equipes no setor.

Durante as visitas, o discente é apresentado a cada sala e nesse momento é possível visualizar tanto a estrutura local quanto o cliente à beira leito e as tecnologias da qual faz uso, como respiradores, monitores, entre outros. É possível perceber nitidamente a importância da organização da sala, da gestão de enfermagem e os equipamentos ali dispostos e em uso de acordo com a gravidade de cada um deles. Ao adentrar no setor foi possível perceber ainda certa dispersão da equipe de enfermagem, que executava 0 momento da visita apenas tarefas rotineiras, onde, ao indagar a situação específica de uma cliente, uma das profissionais não apresentou resposta à questão.

Nesse momento, na percepção do discente, que está ainda apenas vivenciando uma aproximação com o cenário, é impactante constatar a incipiência de informações, gestão organizacional, práxis e previsão do corpo de enfermagem ali presente. Este fato se torna um contraponto à formação acadêmica uma vez que nela aprende-se que o gerenciamento é de suma importância na construção de profissionais capacitados bem como da qualidade da assistência.

Considerando que uma das funções privativas do enfermeiro é a gerência da enfermagem e que tal processo garante todo fluxo da assistência prestada, inclusive da equipe multiprofissional, a visita técnica permite ao discente minimamente elaborar um filtro no que tange às rotinas e cotidiano da equipe de enfermagem, de que forma essas equipes atuam e forma executam suas tarefas.

Além disso, a realização da visita leva o discente a refletir não somente em relação ao ambiente de trabalho e suas práticas, mas também ao tipo de profissional que se seja ser, considerando que a enfermagem é uma profissão que merece seu devido destaque, notoriedade e autonomia e que isso depende fundamentalmente da equipe e seu gerenciamento (DUEÑAS CVM, et al., 2015).

\section{DISCUSSÃO}

\section{O processo de formação do enfermeiro}

Durante o desenvolvimento do processo de formação do enfermeiro na graduação, o discente perpassa por uma série de sentimentos e sensações que podem variar desde a insegurança diante do novo até o peso de pela primeira encontrar-se diante de situações e percepções que o levam a compreender as responsabilidades imbuídas no trabalho do enfermeiro. Percebe-se ainda como fator que potencializa a insegurança, a falta do domínio e prática das atividades que esse discente ainda não possui. Ainda no que tange a esse processo de formação, outra dificuldade encontrada tem relação direta com a imprecisão conceitual que transpõe o campo da saúde e retrata diretamente o contexto da formação e atenção desenvolvida pelos profissionais de saúde nas rotinas institucionais (PINHEIRO DGM, et al., 2015).

O ensino das competências ao discente deve permear-se a partir do estímulo ao desenvolvimento do raciocínio crítico e reflexivo. Deve-se implementar condutas na prática docente que contenham os princípios que conduzam o discente a assumir a corresponsáveis pela sua aprendizagem. $O$ docente, enquanto facilitador deste processo deverá criar e estimular as experiências educativas inerentes à saúde na formação desse discente. Para o desenvolvimento efetivo dessas competências é necessário expandir essa discussão 
para que mudanças sejam adaptadas ao contexto da formação brasileira, de modo a aumentar as possibilidades do desenvolvimento efetivo dessas ações (TUSSET D, et al., 2015).

Concomitante a isso, percebe-se ainda que muitas vezes, na formação do enfermeiro, é possível identificar um modelo biologicista e hospitalocêntrico, pautado em práticas fracionadas evidenciando uma separação entre teoria e prática que influencia diretamente e negativamente na construção desse profissional. Considerar posturas possíveis para mudanças positivas desse contexto é pensar novas formas de ensino que agreguem o entendimento ampliado da saúde e da educação por esse discente de forma que ele perceba a articulação entre as mesmas. Proporcionar novos espaços e momentos de aprendizagem e experienciar práticas integrais que levem à ampliação das competências vivenciadas pelo discente da graduação em enfermagem são alguns desses momentos de congraçamento (ALMEIDA JR, 2013).

Entretanto, analisando o contexto do processo de formação do discente em consonância ao que de fato é vivido no momento prático, percebe-se que infelizmente, nem tudo que é vivenciado pode ser considerado experiência. Essa afirmativa indica que algumas vivências são "apenas" experimentadas pelo sujeito, e muitas vezes não proporcionam o momento desejado ou a expectativa criada sobre o mesmo (SILVA KLD, et al., 2018).

\section{A visita técnica como método de ensino-aprendizagem}

A visita técnica como método de ensino-aprendizagem, permite vivenciar a experiência de estar no futuro ambiente de trabalho através da observação das abordagens, rotinas empregadas, o cuidado, o contato direto com o paciente, a empatia e a postura do profissional diante das adversidades. Para que o processo de ensino-aprendizagem gere a construção do raciocínio crítico reflexivo baseado nos conhecimentos teóricos fornecidos ao discente, as instituições de ensino superior, bem como seus docentes, devem reformular sua metodologia bem como sua postura de ensino bancário tradicional. É necessário mais que apenas transmitir ideias. É preciso buscar novas metodologias e formas de ensino, a fim de apropriar-se das novas perspectivas educacionais fazendo a associação com a realidade atual da sociedade do conhecimento (PAIM AS, et al., 2015).

Considerando o preparo exigido dos profissionais da saúde diante das necessidades cotidianas, e sendo a aprendizagem um processo de construção diária, que exige do discente a capacidade de ação e reflexão muitas vezes imediatas, faz-se necessário que os docentes responsáveis por essa construção da formação desse profissional se aproprie, não somente ao que tange a dinâmica teória-prática. Esse docente precisará reconhecer e se empoderar de seu do papel social enquanto mediador desse processo de construção (DIAS $E P$, et al., 2014).

Nesse sentido, a prática docente precisa, entre outras coisas, instaurar a dúvida, trabalhar com as incertezas e promover a busca de compreensão integrativa dos temas a serem abordados com os discentes, sendo a visita técnica um dos cenários ideiais para desenvolvimento dessa prática (CRUZ RAO, et al., 2017).

Dentre as inúmeras dificuldades encontradas pelo discente durante a graduação, uma delas transita diretamente pela influência decorrente das relações interpessoais vividas em seu processo de formação. Essa influência é principalmente relacionada com a experiência da realização das atividades práticas, dentre elas, a visita técnica. As dificuldades frequentemente enfrentadas por esse discente referem-se principalmente aos processos de relacionamento e comunicação com as equipes presentes nos ambientes de trabalho visitados, com os docentes e com os usuários do sistema de saúde, e além disso, associa-se a essa experiência o medo e ansiedade oriundos das situações imprevisíveis que ocorrem nas visitas. O momento da visita técnica é, portanto, vivenciado pelo discente como a oportunidade de adquirir e agregar os conhecimentos obtidos aos valores da autoconfiança no intuito de torná-los claramente mais preparados e confortáveis para lidar com as práticas relacionadas ao processo de formação (DIAS EP, et al., 2014).

\section{O Sistema Único de Saúde e o ensino}

A história da formação em saúde no Brasil é marcada pela desarticulação entre o ensino e as necessidades sociais de saúde. A problemática em torno da capacidade do sistema de saúde em responder às 
necessidades de saúde entrelaça-se ao desafio do sistema educacional em formar profissionais capazes de intervir nessa direção O processo de desestruturação do SUS é um fato evidente na realidade da saúde no país (AMARAL MCS, et al., 2014). Quando o discente, que permanece durante anos no ambiente universitário onde tudo está em devida ordem, percebe o contraste existente entre as práticas ensinadas na graduação e as efetivamente realizadas no cenário, surge um conflito. Nesse momento, fica latente a percepção de que a defesa pelos princípios de universalidade, equidade e integralidade do SUS não estão sendo devidamente cumpridas.

Em um panorama geral, percebe-se que o SUS sofre uma série de adversidades e seu processo de funcionamento perpassa por inúmeras questões que vão desde interesses econômicos e financeiros ligados à operadoras de planos de saúde e empresas de publicidade até as indústrias farmacêuticas e de equipamentos médico-hospitalares. Com relação ao ensino, apesar de a Constituição proclamar a saúde como direito de todos e dever do Estado, esse preceito não tem assegurado as condições objetivas para a sustentabilidade econômica e científico-tecnológica do SUS, comprometendo sobremaneira o processo de formação e ensino dos acadêmicos nas diversas áreas da saúde e comprometendo diretamente a qualidade do cuidado e o acesso universal e igualitário às ações e serviços de saúde (PAIM JS, 2018).

O momento atual do SUS reflete na diminuição do potencial de ensino-aprendizagem e na assistência à população. Diante disso, para que ocorra a aprendizagem efetiva do discente, faz-se necessário que o sistema forneça recursos estruturais, materiais e humanos, além de cenários com os devidos investimentos e equipes integradas e comprometidas. Assim, a qualificação do serviço e as mudanças nas políticas de saúde promoveriam melhorias na qualidade da assistência em saúde garantindo também a repercussão positiva para o aprendizado na prática (ADLER MS e GALLIAN DMC, 2016).

\section{CONSIDERAÇÕES FINAIS}

A experiência vivida mostrou que é possível desenvolver competências e habilidades no ensino clínico prático em alta complexidade da graduação a partir das visitas técnicas supervisionadas. Foi possível trabalhar uma série de temáticas que permitiram aos discentes aprimorarem o conteúdo teórico-prático para o desenvolvimento dessas competências. Cabe salientar que, em muitos momentos, foi possível constatar as fragilidades existentes no processo de trabalho de uma unidade sob gestão do SUS, fator que dificulta a aprendizagem. Uma vez que não existam disponíveis todos os recursos, insumos e demais materiais necessários à realização de procedimentos através das técnicas corretas ensinadas na graduação, vivenciar esse cenário pode, muitas vezes, despertar no discente o sentimento de insegurança, incerteza e até mesmo frustração diante da percepção de que essas técnicas não são executadas com o rigor esperado. Este fato corrobora e interfere diretamente na experiência de aprendizado. Conclui-se, portanto, que, a visita técnica permitir ao discente vivenciar experiências positivas e negativas, e esse contexto aprimora profundamente suas dimensões intelectuais, relacionais e afetivas do cuidado, favorecendo seu processo de formação e construção de sua dinâmica enquanto futuro profissional.

\section{REFERÊNCIAS}

1. ADLER MS, GALLIAN DMC. Escola médica e Sistema Único de Saúde (SUS): criação do curso de medicina da Universidade Federal de São Carlos, SP, Brasil (UFSCar) sob perspectiva de docentes e estudantes. Interface (Botucatu). 2018; vol.22, n.64, pp.237-249.

2. ALMEIDA JR. Experiência, acontecimento e educação a partir de Foucault. Filogênese [Internet]. 2013; 6(2):48-62.

3. AMARAL MCS, et al. O ensino de Educação Popular em Saúde para o SUS: experiência de articulação entre graduandos de enfermagem e Agentes Comunitários de Saúde. Interface (Botucatu), 2014; v. 18, supl. 2, p. 15471558.

4. BRASIL. Ministério da Educação. Parecer CNE/CES no 1.133/2001, aprovado em 7 de agosto de 2001. Dispõe sobre as Diretrizes Curriculares Nacionais dos Cursos de Graduação em Enfermagem, Medicina e Nutrição [Internet]. Diário Oficial da União. 7 Ago 2001. 
5. COSTA DAS, et al. Diretrizes curriculares nacionais das profissões da Saúde 2001-2004: análise à luz das teorias de desenvolvimento curricular. Interface (Botucatu), 2018; vol.22, n.67, pp.1183-1195.

6. CRUZ RAO, et al. Reflexões à luz da Teoria da Complexidade e a formação do enfermeiro. Rev Bras Enferm, 2017; 70(1): 236-239.

7. DIAS EP, et al. Expectativas de alunos de enfermagem frente ao primeiro estágio em instituições de saúde. Rev. psicopedag., São Paulo, 2014; v. 31, n. 94, p. 44-55.

8. DUEÑAS CVM, et al. Ótica do acadêmico de enfermagem frente ao contato com o paciente hospitalar: discutindo a qualidade do estágio e participação do preceptor. Saber Científico, Porto Velho, 2015; v.4, n. 2, p.55 - 64, dez.

9. HUMEREZ DC, JANKEVICIUS JV. Reflexão sobre a formação das categorias profissionais de saúde de nível superior pós diretrizes curriculares. Brasília, DF: Cofen, 2015.

10. PAIM AS, et al. Métodos de enseñanza utilizados por docentes del curso de enfermería: enfoque en la metodología de investigación. Enferm. Glob, 2015; 14(37): 136-152.

11. PAIM JS. Sistema Único de Saúde (SUS) aos 30 anos. Ciênc. saúde coletiva. 2018; vol.23, n.6, pp.1723-1728.

12. PARANHOS VD, MENDES MMR. Currículo por competência e metodologia ativa: percepção de estudantes de enfermagem. Rev. Latino-Am. Enfermagem, 2010; v.18, n.1, p. 109-115.

13. PINHEIRO DGM, et al. Health promotion competencies: challenges of formation. Saúde Soc, 2015; 24(1):180-8.

14. SILVA KLD, et al. Entre experimentações e experiências: desafios para o ensino das competências para a promoção da saúde na formação do enfermeiro. Interface - Comunicação, Saúde, Educação, 2018; 22(67): 1209-1220.

15. TUSSET D, et al. Analysis of the health promotion competencies from the official document and the speeches of the actors that implement the Health School Program in Federal District. Tempus Actas Saúde Colet., 2015; 9(1):189204. 